\title{
Evaluation of selected parameters of the coagulation system during the perioperative period in patients undergoing endoscopic surgery of the paranasal sinuses
}

Joanna Urbaniak, Kalina Owczarek, Jarosław Miłoński, Piotr Pietkiewicz, Anna Jałocha-Kaczka, Jurek Olszewski

Department of Otolaryngology, Laryngological Oncology, Audiology and Phoniatrics, Medical University of Lodz, Lodz, Poland

Submitted: 8 December 2016

Accepted: 19 September 2017

Arch Med Sci 2020; 16 (6): 1336-1345

DOI: https://doi.org/10.5114/aoms.2017.72544

Copyright (c 2018 Termedia \& Banach

\section{Abstract}

Introduction: The aim of the study was to evaluate selected parameters of the coagulation system during the perioperative period in patients undergoing endoscopic sinus surgery.

Material and methods: The study involved 121 patients: group I - 42 patients who did not receive anticoagulatory or antiplatelet medications, qualified for endoscopic sinus surgery under total intravenous anaesthesia (TIVA); group II - 40 patients who received in the perioperative period low-molecular-weight heparins, qualified for endoscopic sinus surgery under TIVA; group III - 39 patients diagnosed according to a schedule, due to vertigo or loss of hearing. All the patients received a full laryngological examination and detailed audiological and otoneurological diagnostics, and examination of selected haemostatic parameters before the surgery/diagnostics.

Results: The analysis of concentrations of coagulation parameters in groups I and II revealed a statistically significantly higher international normalized ratio value before surgery $(\mathrm{I}-1.11 ; \mathrm{I}-1.08)$ and $48 \mathrm{~h}$ following surgery $(I-1.15 ; \mid I-1.10)$ in group I. The concentration of coagulation factor VII in the study patients was considerably higher in group I for all three measurements $(481.93 ; 443.13 ; 486.02)$. The concentration of fibrinogen (coagulation factor I) was significantly lower in group I before surgery (3.2) and at $6 \mathrm{~h}$ after surgery (2.84). A significantly lower level of von Willebrand factor was found in group I before surgery (2.94). Comparing test results of groups I and III, who did not receive antiaggregants, statistically significant differences were observed in both tests for factors VII and VIII.

Conclusions: Concentrations of von Willebrand factor and prothrombin revealed statistically significant differences in between groups.

Key words: intranasal surgery, low-molecular-weight heparin, antiplatelet drugs, blood coagulation factors, intravenous anaesthesia

\section{Introduction}

Over the last 30 years, minimally invasive, endoscopic surgery of the paranasal sinuses (functional endoscopic sinus surgery - FESS) has almost completely replaced open surgery of the paranasal sinuses due to chronic inflammation, with or without polyps, non-responsive to conservative treatment. Presently, FESS is the method of choice in the therapy of chronic sinusitis posing difficulties to conservative treatment. The high

\section{Corresponding author:} Prof. Jurek Olszewski MD, PhD Department of Otolaryngology, Laryngological Oncology, Audiology and Phoniatrics Medical University of Lodz

113 Żeromskiego St 90-549 Lodz, Poland E-mail: jurek.olszewski@ umed.lodz.pl 
effectiveness of FESS results in a constant evolution in surgical techniques, as well as preoperative, perioperative and post-operative treatments [1, 2].

Due to the ongoing improvement in endoscopic techniques, they are increasingly often used for the surgical therapy of tumours of the nasal cavities and paranasal sinuses. This is associated with the advanced age of patients qualified for endoscopic procedures, the related systemic burden to the patients, and the increasing quantity of medications used, including those which affect the coagulation system and fibrinolysis [3].

Considering the possible predisposing factors for post-FESS complications, one cannot ignore the fact that, apart from anatomic abnormalities in the operated area, local progression of the disease or re-operation, important risk factors for complications include age, general health and medications used $[4,5]$.

The most common complications are as follows: leakage of cerebrospinal fluid, damage to the orbit and haemorrhage, which often require transfusion of blood products $[6,7]$. One reason for excessive bleeding during FESS, apart from the injury resulting from the procedure, is coagulation disorders. The effectiveness of coagulation is determined by vascular, platelet and coagulation factors. The vascular factors include hereditary haemorrhagic telangiectasias (e.g. Osler-Weber-Rendu disease), which affects mostly the face and nose.

The platelet factor is associated with a reduced number of platelets, or disorders regarding their aggregation, and it includes idiopathic thrombocytopenic purpura, renal or hepatic failure, and the use of acetylsalicylic acid (Aspirin, Acard, Polocard).

Disorders due to deficiencies in coagulation factors are usually related to the use of anticoagulants (warfarin, heparin), or cause hereditary diseases, such as haemophilia or von Willebrand disease [8].

As society ages, the risk of haemorrhage associated with anticoagulatory/anti-platelet therapy increases. Indications for these therapies primarily include internal, cardiac or neurological conditions, and they include venous thrombotic embolism, atrial fibrillation, cerebrovascular and cardiovascular incidents [9-12].

In North America each year the above therapy needs to be stopped due to planned surgery [13-16]. To ensure patient safety, a bridge therapy is introduced, i.e. anticoagulants and antiplatelet medications are replaced with low-molecular-weight heparin during the perioperative period [17, 18].

Low-molecular-weight heparin forms a complex with antithrombin III and blocks coagulation factor X; most importantly, it also blocks thrombin, thus preventing activation of the coagulation sys- tem without increasing the risk of intraoperative and postoperative bleeding. It is a popular medication, due to the very good safety profile, possible outpatient therapy, and no need to monitor the international normalised ratio (INR) [19-21].

The aim of the study is to evaluate selected factors of the coagulation system during the perioperative period in patients undergoing endoscopic surgery of the paranasal sinuses.

\section{Material and methods}

The study involved 121 patients of the Department of Otolaryngology, Laryngological Oncology, Audiology and Phoniatrics at the Medical University of Lodz, Military Medical Academy Teaching Hospital. They were divided into 3 groups:

- Group I - 42 patients, including 12 females and 30 males, aged $20-70$ years (mean age: 33.68 \pm 9.56 years), who did not receive antithrombotic or antiplatelet medications, qualified for endoscopic treatment of paranasal sinuses (endoscopic ethmoidectomy, revision of maxillary, frontal and sphenoid sinuses) under TIVA anaesthesia, or who had discontinued medications affecting the haemostatic system over 10 days prior to the study.

- Group II - 40 patients, including 12 females and 28 males, aged 33-74 years (mean age: 58.38 \pm 9.71 years), who received in the perioperative period low-molecular-weight heparins according to individual cardiac or internist recommendations, qualified for endoscopic treatment of paranasal sinuses (endoscopic ethmoidectomy, revision of maxillary, frontal and sphenoid sinuses) under TIVA.

- Group III - 39 patients, including 16 females and 23 males, aged $29-77$ years (mean age: $57.62 \pm 14.01$ ), undergoing elective diagnostics due to vertigo or hearing loss, 19 of the patients ( 7 females and 12 males) using antiplatelet medications according to individual cardiac or internist recommendations, and 20 patients ( 9 females and 11 males) either not using those medications, or having discontinued medications affecting the haemostatic system over 10 days prior to the study.

The patients included in the study received a complete laryngological examination, as well as detailed audiological and otoneurological diagnostics. In groups I and II, laboratory tests were performed to qualify the patients for laryngological surgery. Moreover, selected parameters of the haemostatic system were examined in all patients: assessment of the levels of fibrinogen, prothrombin, coagulation factors VII and VIII, and von Willebrand factor, and assessment of activated partial thromboplastin time (APTT) and prothrombin time (PT) (before surgery/diagnostics, after 6 and $48 \mathrm{~h}$ following surgery). 
The exclusion criteria included: coagulopathy, cardiovascular insufficiency, renal failure, and neoplasms.

The study was approved by the Bioethical Committee of the Medical University of Lodz - approval no. RNN/144/15/KE of 19.09.2015.

\section{Statistical analysis}

Statistical significance of the results was assumed at $p<0.05$. The Stata/Special Edition version 12.1 (StataCorp LP, College Station, Texas, USA) software was used for statistical analysis.

Statistical tests used for tables: analysis of variance with repeated measures (when the distribution of variables met the assumptions of normality and sphericity, and variances were homogeneous); Friedman's test of ANOVA on ranks (when the distribution did not meet the assumptions of normality and sphericity, and the variances were not homogeneous).

Statistical tests used for figures: univariate analysis of variance without repetition (when the distribution of variables met the assumptions of normality and variances were homogeneous) or Mann-Whitney $U$ test (if the distribution did not meet the assumptions of normality, and the variances were not homogeneous).

\section{Results}

In group I, the mean values for the coagulation testing results were as follows (Tables I-III): before surgery PT - $12.76 \mathrm{~s}$, INR - 1.11, APTT - $1.01 \mathrm{~s}$; $6 \mathrm{~h}$ after surgery: PT - $13.19 \mathrm{~s}$, INR - 1.14, APTT 0.99 s; 48 h after surgery: PT - 13.26 s, INR -1.15 , APTT $-1.02 \mathrm{~s}$. The observed differences were statistically significant.

In group II, the mean values for the coagulation testing results were as follows (Tables I-III): be- fore surgery PT - $12.38 \mathrm{~s}$, INR - 1.08, APTT - $1.02 \mathrm{~s}$; $6 \mathrm{~h}$ after surgery: PT $-12.97 \mathrm{~s}$, INR -1.12 , APTT $1.01 \mathrm{~s} ; 48 \mathrm{~h}$ after surgery: PT - $12.77 \mathrm{~s}$, INR - 1.10, APTT $-1.02 \mathrm{~s}$, and the observed differences for PT and INR were statistically significant.

In group III, the mean values for the coagulation testing results were as follows (Tables I-III): before surgery PT - $13.23 \mathrm{~s}$, INR - 1.13, APTT 0.99 s; 24 h after surgery: PT -13.33 s, INR -1.15 , APTT - $1.01 \mathrm{~s}$. The observed differences were not statistically significant (Tables I-III).

The levels of selected coagulation factors were as follows (Table IV): for group I before surgery the concentration of factor VII - $481.93 \mathrm{ng} / \mathrm{ml}$, factor VIII - $92.09 \mathrm{ng} / \mathrm{ml}$, von Willebrand factor $2.94 \mathrm{mU} / \mathrm{ml}$, protein $\mathrm{C}-16.01 \mathrm{mg} / \mathrm{ml}$, fibrinogen - $3.2 \mathrm{~g} / \mathrm{ml}$, and prothrombin - $169.23 \mathrm{ng} / \mathrm{ml}$; $6 \mathrm{~h}$ after surgery: concentration of factor VII - 443.13 $\mathrm{ng} / \mathrm{ml}$, factor VIII - $80.49 \mathrm{ng} / \mathrm{ml}$, von Willebrand factor - $3.02 \mathrm{mU} / \mathrm{ml}$, protein C - 16.42, fibrinogen $-2.84 \mathrm{~g} / \mathrm{ml}$, and prothrombin $-146.28 \mathrm{ng} / \mathrm{ml}$; $48 \mathrm{~h}$ after surgery: concentration of factor VII $486.02 \mathrm{ng} / \mathrm{ml}$, factor VIII - $113.72 \mathrm{ng} / \mathrm{ml}$, von Willebrand factor - $3.66 \mathrm{mU} / \mathrm{ml}$, protein C - 18.48, fibrinogen - $4.24 \mathrm{~g} / \mathrm{ml}$, and prothrombin - 182.13 $\mathrm{ng} / \mathrm{ml}$. Differences in the results were statistically significant for factor VII, factor VIII, von Willebrand factor, fibrinogen and protein $C$.

The levels of selected coagulation factors were as follows (Table V): for group II before surgery the concentration of factor VII - $213.71 \mathrm{ng} / \mathrm{ml}$, factor VIII-99.74 ng/ml, von Willebrand factor $-3.82 \mathrm{mU} /$ $\mathrm{ml}$, protein $\mathrm{C}-17.22 \mathrm{mg} / \mathrm{ml}$, fibrinogen $-4.0 \mathrm{~g} / \mathrm{ml}$, and prothrombin - $175.69 \mathrm{ng} / \mathrm{ml}$; $6 \mathrm{~h}$ after surgery: concentration of factor VII - $194.33 \mathrm{ng} / \mathrm{ml}$, factor VIII - $95.26 \mathrm{ng} / \mathrm{ml}$, von Willebrand factor $3.71 \mathrm{mU} / \mathrm{ml}$, protein $\mathrm{C}-17.19 \mathrm{mg} / \mathrm{ml}$, fibrinogen $3.68 \mathrm{~g} / \mathrm{ml}$, and prothrombin - $164.16 \mathrm{ng} / \mathrm{ml} ; 48 \mathrm{~h}$

Table I. Descriptive statistics for prothrombin time in examined patients, according to study group and time of measurement

\begin{tabular}{|c|c|c|c|c|c|c|c|c|}
\hline \multirow[t]{2}{*}{ Group } & \multirow{2}{*}{$\begin{array}{c}\text { Sample } \\
\text { size } \\
(n)\end{array}$} & \multirow{2}{*}{$\begin{array}{c}\text { Time } \\
\text { of measurement }\end{array}$} & \multicolumn{5}{|c|}{ Statistical parameter } & \multirow{2}{*}{$\begin{array}{l}\text { Statistical } \\
\text { significance } \\
\text { of difference }\end{array}$} \\
\hline & & & $M$ & $\mathrm{Me}$ & SD & $95 \% \mathrm{Cl}$ & Min.-max. & \\
\hline \multirow[t]{3}{*}{ I } & 40 & Before surgery & 12.76 & 12.75 & 0.94 & $12.45-13.05$ & $10.50-14.80$ & $p<0.001$ \\
\hline & & $6 \mathrm{~h}$ after surgery & 13.19 & 13.00 & 0.87 & $12.91-13.47$ & $11.90-16.20$ & \\
\hline & & $48 \mathrm{~h}$ after surgery & 13.26 & 13.25 & 1.04 & $12.93-13.59$ & $11.30-16.70$ & \\
\hline \multirow[t]{3}{*}{ II } & 40 & Before surgery & 12.38 & 12.30 & 0.69 & $12.15-12.60$ & $10.80-14.50$ & $p<0.001$ \\
\hline & & $6 \mathrm{~h}$ after surgery & 12.97 & 12.95 & 0.70 & $12.74-13.19$ & $11.30-14.80$ & \\
\hline & & $48 \mathrm{~h}$ after surgery & 12.77 & 12.75 & 0.72 & $12.54-13.00$ & $11.10-14.80$ & \\
\hline \multirow[t]{2}{*}{ III } & 39 & Before diagnostics & 13.23 & 13.00 & 2.07 & $12.56-13.90$ & $11.50-22.20$ & $p=0.254$ \\
\hline & & $24 \mathrm{~h}$ after diagnostics & 13.33 & 12.90 & 2.12 & $12.65-14.02$ & $11.70-22.40$ & \\
\hline
\end{tabular}

Prothrombin time, PT expressed in seconds (s). Statistical parameters: $M-$ mean value, Me - median value, SD - standard deviation, 95\% Cl-95\% confidence interval. 
Table II. Descriptive statistics for international normalised ratio in examined patients, according to study group and time of measurement

\begin{tabular}{|c|c|c|c|c|c|c|c|}
\hline \multirow[t]{2}{*}{ Group } & \multirow{2}{*}{$\begin{array}{c}\text { Time } \\
\text { of measurement }\end{array}$} & \multicolumn{5}{|c|}{ Statistical parameter } & \multirow{2}{*}{$\begin{array}{l}\text { Statistical } \\
\text { significance } \\
\text { of difference }\end{array}$} \\
\hline & & $M$ & $\mathrm{Me}$ & SD & $95 \% \mathrm{Cl}$ & Min.-max. & \\
\hline \multirow[t]{3}{*}{ । } & Before surgery & 1.11 & 1.11 & 0.07 & $1.09-1.13$ & $0.95-1.25$ & $p<0.001$ \\
\hline & $6 \mathrm{~h}$ after surgery & 1.14 & 1.14 & 0.06 & $1.12-1.16$ & $1.05-1.37$ & \\
\hline & $48 \mathrm{~h}$ after surgery & 1.15 & 1.13 & 0.08 & $1.12-1.17$ & $1.02-1.42$ & \\
\hline \multirow[t]{3}{*}{ II } & Before surgery & 1.08 & 1.08 & 0.06 & $1.06-1.09$ & $0.95-1.21$ & $p<0.001$ \\
\hline & $6 \mathrm{~h}$ after surgery & 1.12 & 1.12 & 0.05 & $1.10-1.14$ & $0.98-1.24$ & \\
\hline & $48 \mathrm{~h}$ after surgery & 1.10 & 1.11 & 0.06 & $1.09-1.12$ & $0.96-1.24$ & \\
\hline \multirow[t]{2}{*}{ III } & Before diagnostics & 1.13 & 1.09 & 0.17 & $1.08-1.18$ & $1.00-1.84$ & $p=0.027$ \\
\hline & $24 \mathrm{~h}$ after diagnostics & 1.15 & - & 0.17 & $1.09-1.20$ & $1.03-1.90$ & \\
\hline
\end{tabular}

International normalized ratio (INR) displayed as a coefficient (no unit of measurement). Study group sizes: See Table I. Statistical parameters: See Table I.

Table III. Descriptive statistics for activated partial thromboplastin time in examined patients, according to study group and time of measurement

\begin{tabular}{|c|c|c|c|c|c|c|c|}
\hline \multirow[t]{2}{*}{ Group } & \multirow{2}{*}{$\begin{array}{c}\text { Time } \\
\text { of measurement }\end{array}$} & \multicolumn{5}{|c|}{ Statistical parameter } & \multirow{2}{*}{$\begin{array}{l}\text { Statistical } \\
\text { significance } \\
\text { of difference }\end{array}$} \\
\hline & & $M$ & Me & SD & $95 \% \mathrm{Cl}$ & Min.-max. & \\
\hline \multirow[t]{3}{*}{1} & Before surgery & 1.01 & 1.00 & 0.12 & $0.97-1.04$ & $0.80-1.30$ & $p=0.027$ \\
\hline & $6 \mathrm{~h}$ after surgery & 0.99 & 1.00 & 0.13 & $0.95-1.03$ & $0.80-1.30$ & \\
\hline & $48 \mathrm{~h}$ after surgery & 1.02 & 1.00 & 0.13 & $0.98-1.06$ & $0.80-1.30$ & \\
\hline \multirow[t]{3}{*}{ II } & Before surgery & 1.02 & 1.00 & 0.13 & $0.98-1.06$ & $0.80-1.40$ & $p=0.331$ \\
\hline & $6 \mathrm{~h}$ after surgery & 1.01 & 1.00 & 0.12 & $0.97-1.04$ & $0.80-1.40$ & \\
\hline & $48 \mathrm{~h}$ after surgery & 1.02 & 1.00 & 0.11 & $0.99-1.06$ & $0.80-1.30$ & \\
\hline \multirow[t]{2}{*}{ III } & Before diagnostics & 0.99 & 0.00 & 0.14 & $0.95-1.04$ & $0.80-1.50$ & $p=0.031$ \\
\hline & $24 \mathrm{~h}$ after diagnostics & 1.01 & 1.00 & 0.14 & $0.96-1.06$ & $0.80-1.30$ & \\
\hline
\end{tabular}

Activated partial thromboplastin time, APTT expressed in seconds (s). Study group sizes: See Table I. Statistical parameters: See Table I.

after surgery: concentration of factor VII - 195.53 $\mathrm{ng} / \mathrm{ml}$, factor VIII - $129.57 \mathrm{ng} / \mathrm{ml}$, von Willebrand factor - $4.41 \mathrm{mU} / \mathrm{ml}$, protein C - $18.97 \mathrm{mg} / \mathrm{ml}$, fibrinogen - $4.56 \mathrm{~g} / \mathrm{ml}$, and prothrombin - 175.56 $\mathrm{ng} / \mathrm{ml}$. The differences in the results were statistically significant for factors VII and VIII, von Willebrand factor, fibrinogen and protein $\mathrm{C}$.

In group III the mean coagulation test results were as follows (Table VI): before the diagnostics concentration of factor VII - $247.74 \mathrm{ng} / \mathrm{ml}$, factor VIII - $132.16 \mathrm{ng} / \mathrm{ml}$, von Willebrand factor - $3.01 \mathrm{mU} /$ $\mathrm{ml}$, protein C $-17.95 \mathrm{mg} / \mathrm{ml}$, fibrinogen $-3.97 \mathrm{~g} / \mathrm{ml}$, and prothrombin $-168.7 \mathrm{ng} / \mathrm{ml} ; 24 \mathrm{~h}$ after the diagnostics: concentration of factor VII $-230.05 \mathrm{ng} / \mathrm{ml}$, factor VIII - $146.28 \mathrm{ng} / \mathrm{ml}$, von Willebrand factor $3.21 \mathrm{mU} / \mathrm{ml}$, protein C - $17.39 \mathrm{mg} / \mathrm{ml}$, fibrinogen $3.69 \mathrm{~g} / \mathrm{ml}$, and prothrombin - $156.11 \mathrm{ng} / \mathrm{ml}$. The differences in the results were statistically significant for factor VII, factor VIII and prothrombin.
The comparison of the results of the international normalised ratio INR in groups I and II revealed significantly higher values in group I in the tests performed before the surgery and $48 \mathrm{~h}$ after the surgery (Figure 1).

A statistically significant correlation was also observed in the comparison of coagulation factor VII in groups I and II. The values were statistically significantly higher in group I than in group II in all three tests, before and after the surgery (Figure 2).

The comparison of fibrinogen concentration in groups I and II demonstrated that the levels of fibrinogen in group I were statistically significantly lower than in group II in both the test performed before the surgery and $6 \mathrm{~h}$ after the procedure (Figure 3).

The concentration of von Willebrand factor was also statistically significantly lower in group I than in group II, but only in the test conducted before 
Table IV. Descriptive statistics for group I of the level of coagulation factors VII and VIII, fibrinogen, protein C, von Willebrand factor and prothrombin in examined patients, according to time of measurement

\begin{tabular}{|c|c|c|c|c|c|c|c|}
\hline \multirow{2}{*}{$\begin{array}{l}\text { Examined } \\
\text { factor }\end{array}$} & \multirow{2}{*}{$\begin{array}{c}\text { Time } \\
\text { of measurement }\end{array}$} & \multicolumn{5}{|c|}{ Statistical parameter } & \multirow{2}{*}{$\begin{array}{l}\text { Statistical } \\
\text { significance } \\
\text { of difference }\end{array}$} \\
\hline & & M & Me & SD & $95 \% \mathrm{Cl}$ & Min.-max. & \\
\hline \multirow{3}{*}{$\begin{array}{l}\text { Factor VII } \\
{[\mathrm{ng} / \mathrm{ml}]}\end{array}$} & Before surgery & 481.93 & 315.41 & 413.33 & $349.74-614.11$ & $72.31-1562.52$ & \multirow[t]{3}{*}{$p<0.001$} \\
\hline & $6 \mathrm{~h}$ after surgery & 44.13 & 245.40 & 422.29 & $308.08-578.19$ & $66.08-1790.04$ & \\
\hline & $48 \mathrm{~h}$ after surgery & 486.02 & 286.70 & 528.40 & $317.03-655.01$ & $67.86-2856.31$ & \\
\hline \multirow{3}{*}{$\begin{array}{l}\text { Factor VIII } \\
{[\mathrm{ng} / \mathrm{ml}]}\end{array}$} & Before surgery & 92.09 & 73.67 & 71.48 & $69.23-114.95$ & $13.39-297.78$ & \multirow[t]{3}{*}{$p<0.001$} \\
\hline & $6 \mathrm{~h}$ after surgery & 80.49 & 56.61 & 55.83 & $62.63-98.35$ & $15.86-209.03$ & \\
\hline & $48 \mathrm{~h}$ after surgery & 113.72 & 101.64 & 70.07 & $91.31-136.13$ & $15.70-292.85$ & \\
\hline \multirow{3}{*}{$\begin{array}{l}\text { Fibrinogen } \\
{[\mathrm{g} / \mathrm{ml}]}\end{array}$} & Before surgery & 3.20 & 3.00 & 1.05 & $2.86-3.53$ & $1.87-7.65$ & \multirow[t]{3}{*}{$p<0.001$} \\
\hline & $6 \mathrm{~h}$ after surgery & 2.84 & 2.79 & 0.65 & $2.63-3.05$ & $1.76-5.79$ & \\
\hline & $48 \mathrm{~h}$ after surgery & 4.24 & 3.95 & 1.23 & $3.85-4.63$ & $2.36-7.84$ & \\
\hline \multirow{3}{*}{$\begin{array}{l}\text { Protein C } \\
{[\mathrm{mg} / \mathrm{ml}]}\end{array}$} & Before surgery & 16.01 & 15.87 & 4.45 & $14.59-17.44$ & $9.07-29.31$ & \multirow[t]{3}{*}{$p<0.001$} \\
\hline & $6 \mathrm{~h}$ after surgery & 16.42 & 15.29 & 5.58 & $14.64-18.21$ & $9.15-33.77$ & \\
\hline & $48 \mathrm{~h}$ after surgery & 18.48 & 18.47 & 5.12 & $16.84-20.12$ & $9.90-31.42$ & \\
\hline \multirow{3}{*}{$\begin{array}{l}\text { Von } \\
\text { Willebrand } \\
\text { factor } \\
{[\mathrm{mU} / \mathrm{ml}]}\end{array}$} & Before surgery & 2.94 & 2.70 & 1.80 & $2.37-3.52$ & $0.65-8.72$ & \multirow[t]{3}{*}{$p=0.013$} \\
\hline & $6 \mathrm{~h}$ after surgery & 3.02 & 3.05 & 1.94 & $2.40-3.64$ & $0.37-8.59$ & \\
\hline & $48 \mathrm{~h}$ after surgery & 3.66 & 3.34 & 2.31 & $2.92-4.40$ & $0.64-9.05$ & \\
\hline \multirow{3}{*}{$\begin{array}{l}\text { Prothrombin } \\
{[\mathrm{ng} / \mathrm{ml}]}\end{array}$} & Before surgery & 169.23 & 164.94 & 52.17 & $152.54-185.91$ & $96.13-259.62$ & \multirow[t]{3}{*}{$p=0.389$} \\
\hline & $6 \mathrm{~h}$ after surgery & 146.28 & 157.98 & 40.64 & $133.28-159.28$ & $76.23-207.78$ & \\
\hline & $48 \mathrm{~h}$ after surgery & 182.13 & 178.41 & 89.46 & $153.51-210.74$ & $98.49-662.04$ & \\
\hline
\end{tabular}

Number of subjects: $n=40$. Statistical parameters: See Table .

the surgery. In the tests performed after the surgery the differences in fibrinogen concentration were not statistically significant (Figure 4).

No statistically significant correlations were found between groups I and II for APTT, factor VIII, prothrombin and protein $\mathrm{C}$.

The comparison of test results of the group I patients with the group III patients who did not receive antiplatelet medications revealed a statistically significant difference in the concentrations of factors VII and VIII, both before the surgery/ audiological diagnostics, and after the surgery/ diagnostics (Figures 5, 6).

The comparisons of protein $C$ and the von Willebrand factor concentrations before the surgery/diagnostics in group I and group III patients who did not receive antiplatelet medications demonstrated statistically significant differences. In group I the concentration of protein $\mathrm{C}$ and the von Willebrand factor was significantly lower (Figures 7, 8).

The test results for patients from group III were also compared, differentiating between the patients receiving antiplatelet medications and those who did not receive them. For most factors the differences were not statistically significant; only the von Willebrand factor was statistically significantly higher in the group of patients using antiplatelet medications, both before and after the audiological diagnostics (Figure 9).

\section{Discussion}

The number of patients using anticoagulants or antiaggregants who undergo elective surgery is increasing. Traditionally, oral anticoagulation was achieved with vitamin $\mathrm{K}$ antagonists (VKAs). In recent years, newer non-vitamin $\mathrm{K}$ dependent anticoagulants (NOACs) have appeared that provide an effective and comfortable alternative to VKAs [22]. Acetylsalicylic acid is also a common drug in use today [23]. All of them pose a challenge for the operating surgeons, as well as for the attending internists, cardiologists or neurologists. The transition to low-molecular-weight heparin in the perioperative period is now standard.

Understanding the behaviour of the individual parameters of the coagulation system and fibrinolysis should make it possible to monitor the haemostatic system during the perioperative period, and thus introduce early therapy to ensure 
Table V. Descriptive statistics for group II of the level of coagulation factors VII and VIII, fibrinogen, protein C, von Willebrand factor and prothrombin in examined patients, according to time of measurement

\begin{tabular}{|c|c|c|c|c|c|c|c|}
\hline \multirow{2}{*}{$\begin{array}{l}\text { Examined } \\
\text { factor }\end{array}$} & \multirow{2}{*}{$\begin{array}{c}\text { Time } \\
\text { of measurement }\end{array}$} & \multicolumn{5}{|c|}{ Statistical parameter } & \multirow{2}{*}{$\begin{array}{l}\text { Statistical } \\
\text { significance } \\
\text { of difference }\end{array}$} \\
\hline & & M & $\mathrm{Me}$ & SD & $95 \% \mathrm{Cl}$ & Min.-max. & \\
\hline \multirow{3}{*}{$\begin{array}{l}\text { Factor VII } \\
{[\mathrm{ng} / \mathrm{ml}]}\end{array}$} & Before surgery & 213.71 & 175.83 & 200.18 & $149.69-277.73$ & $40.06-1269.85$ & \multirow[t]{3}{*}{$p<0.001$} \\
\hline & $6 \mathrm{~h}$ after surgery & 194.3 & 157.17 & 194.97 & $131.97-256.68$ & $34.81-1224.86$ & \\
\hline & $48 \mathrm{~h}$ after surgery & 195.3 & 162.80 & 184.01 & $136.68-254.38$ & $36.77-1165.21$ & \\
\hline \multirow{3}{*}{$\begin{array}{l}\text { Factor VIII } \\
{[\mathrm{ng} / \mathrm{ml}]}\end{array}$} & Before surgery & 99.74 & 84.22 & 66.01 & $78.63-120.85$ & $14.04-268.21$ & \multirow[t]{3}{*}{$p<0.001$} \\
\hline & $6 \mathrm{~h}$ after surgery & 95.26 & 68.38 & 63.95 & $74.80-115.71$ & $26.72-242.94$ & \\
\hline & 48 h after surgery & 129.57 & 12.73 & 82.17 & $103.29-155.85$ & $2.69-297.78$ & \\
\hline \multirow{3}{*}{$\begin{array}{l}\text { Fibrinogen } \\
{[\mathrm{g} / \mathrm{l}]}\end{array}$} & Before surgery & 4.00 & 3.71 & 1.20 & $3.62-4.39$ & $2.33-8.99$ & \multirow[t]{3}{*}{$p<0.001$} \\
\hline & $6 \mathrm{~h}$ after surgery & 3.68 & 3.52 & 1.19 & $3.30-4.06$ & $2.41-7.37$ & \\
\hline & $48 \mathrm{~h}$ after surgery & 4.56 & 4.57 & 0.97 & $4.25-4.86$ & $2.88-7.07$ & \\
\hline \multirow{3}{*}{$\begin{array}{l}\text { Protein C } \\
{[\mathrm{mg} / \mathrm{l}]}\end{array}$} & Before surgery & 17.22 & 16.97 & 5.15 & $15.57-18.86$ & $9.04-26.18$ & \multirow[t]{3}{*}{$p=0.002$} \\
\hline & $6 \mathrm{~h}$ after surgery & 17.19 & 16.37 & 6.25 & 15.19-19.19 & $6.75-35.42$ & \\
\hline & $48 \mathrm{~h}$ after surgery & 18.97 & 18.99 & 6.09 & $17.02-20.92$ & $9.37-40.62$ & \\
\hline \multirow{3}{*}{$\begin{array}{l}\text { Von } \\
\text { Willebrand } \\
\text { factor } \\
{[\mathrm{mU} / \mathrm{ml}]}\end{array}$} & Before surgery & 3.82 & 3.73 & 1.74 & $3.27-4.38$ & $0.76-7.44$ & \multirow[t]{3}{*}{$p=0.001$} \\
\hline & $6 \mathrm{~h}$ after surgery & 3.71 & 3.41 & 1.83 & $3.12-4.30$ & $0.64-9.66$ & \\
\hline & $48 \mathrm{~h}$ after surgery & 4.41 & 4.56 & 1.72 & $3.86-4.96$ & $0.95-8.70$ & \\
\hline \multirow{3}{*}{$\begin{array}{l}\text { Prothrombin } \\
{[\mathrm{ng} / \mathrm{ml}]}\end{array}$} & Before surgery & 175.69 & 175.89 & 42.36 & $162.14-189.23$ & $92.97-260.80$ & \multirow[t]{3}{*}{$p=0.981$} \\
\hline & $6 \mathrm{~h}$ after surgery & 164.6 & 170.25 & 5.74 & $147.61-180.71$ & $70.50-266.38$ & \\
\hline & $48 \mathrm{~h}$ after surgery & 175.56 & 184.60 & 40.97 & $162.46-188.66$ & $102.00-238.85$ & \\
\hline
\end{tabular}

Number of subjects: $n=40$. Statistical parameters: See Table I.

Table VI. Descriptive statistics for group III of the level of coagulation factors VII and VIII, fibrinogen, protein C, von Willebrand factor and prothrombin in examined patients, according to time of measurement

\begin{tabular}{|c|c|c|c|c|c|c|c|}
\hline \multirow{2}{*}{$\begin{array}{l}\text { Examined } \\
\text { factor }\end{array}$} & \multirow{2}{*}{$\begin{array}{c}\text { Time } \\
\text { of measurement }\end{array}$} & \multicolumn{5}{|c|}{ Statistical parameter } & \multirow{2}{*}{$\begin{array}{l}\text { Statistical } \\
\text { significance } \\
\text { of difference }\end{array}$} \\
\hline & & M & Me & SD & $95 \% \mathrm{Cl}$ & Min.-max. & \\
\hline \multirow[t]{2}{*}{$\begin{array}{l}\text { Factor VII } \\
{[\mathrm{ng} / \mathrm{ml}]}\end{array}$} & $\begin{array}{c}\text { Before } \\
\text { diagnostics }\end{array}$ & 247.74 & 108.19 & 371.30 & $127.38-368.11$ & $47.16-163.66$ & $p<0.001$ \\
\hline & $\begin{array}{c}24 \mathrm{~h} \text { after } \\
\text { diagnostics }\end{array}$ & 230.05 & 111.66 & 323.18 & 125.29 & 48.50 & \\
\hline \multirow[t]{2}{*}{$\begin{array}{l}\text { Factor VIII } \\
{[\mathrm{ng} / \mathrm{ml}]}\end{array}$} & $\begin{array}{c}\text { Before } \\
\text { diagnostics }\end{array}$ & 132.16 & 134.77 & 67.93 & $110.14-154.18$ & $36.41-292.85$ & $p<0.001$ \\
\hline & $\begin{array}{c}24 \mathrm{~h} \text { after } \\
\text { diagnostics }\end{array}$ & 146.28 & 112.02 & 145.38 & 99.16-193.41 & $32.32-898.35$ & \\
\hline \multirow[t]{2}{*}{$\begin{array}{l}\text { Fibrinogen } \\
{[\mathrm{g} / \mathrm{l}]}\end{array}$} & $\begin{array}{c}\text { Before } \\
\text { diagnostics }\end{array}$ & 3.97 & 3.42 & 1.50 & $3.48-4.45$ & $2.20-8.27$ & $p=0.066$ \\
\hline & $\begin{array}{c}24 \mathrm{~h} \text { after } \\
\text { diagnostics }\end{array}$ & 3.69 & 3.41 & 1.25 & $3.29-4.10$ & $1.52-6.54$ & \\
\hline \multirow[t]{2}{*}{$\begin{array}{l}\text { Protein C } \\
{[\mathrm{mg} / \mathrm{l}]}\end{array}$} & $\begin{array}{c}\text { Before } \\
\text { diagnostics }\end{array}$ & 17.95 & 17.42 & 5.11 & $16.29-19.60$ & $10.40-30.56$ & $p=0.257$ \\
\hline & $\begin{array}{c}24 \mathrm{~h} \text { after } \\
\text { diagnostics }\end{array}$ & 17.39 & 16.10 & 4.81 & $15.83-18.95$ & $8.21-29.53$ & \\
\hline \multirow{2}{*}{$\begin{array}{l}\text { Von } \\
\text { Willebrand } \\
\text { factor } \\
{[\mathrm{mU} / \mathrm{ml}]}\end{array}$} & $\begin{array}{c}\text { Before } \\
\text { diagnostics }\end{array}$ & 3.01 & 2.76 & 1.84 & $2.41-3.60$ & $0.66-6.09$ & $p=0.515$ \\
\hline & $\begin{array}{l}24 \mathrm{~h} \text { after } \\
\text { diagnostics }\end{array}$ & 3.21 & 2.81 & 2.03 & $2.56-8.87$ & $0.59-9.90$ & \\
\hline \multirow[t]{2}{*}{$\begin{array}{l}\text { Prothrombin } \\
{[\mathrm{ng} / \mathrm{ml}]}\end{array}$} & $\begin{array}{c}\text { Before } \\
\text { diagnostics }\end{array}$ & 168.70 & 166.41 & 49.64 & 152.61-184.79 & $79.67-300.62$ & $p=0.039$ \\
\hline & $\begin{array}{l}24 \mathrm{~h} \text { after } \\
\text { diagnostics }\end{array}$ & 156.11 & 165.08 & 47.60 & $140.68-171.54$ & $56.90-281.63$ & \\
\hline
\end{tabular}

Number of subjects: $n=39$. Statistical parameters: See Table 1 . 


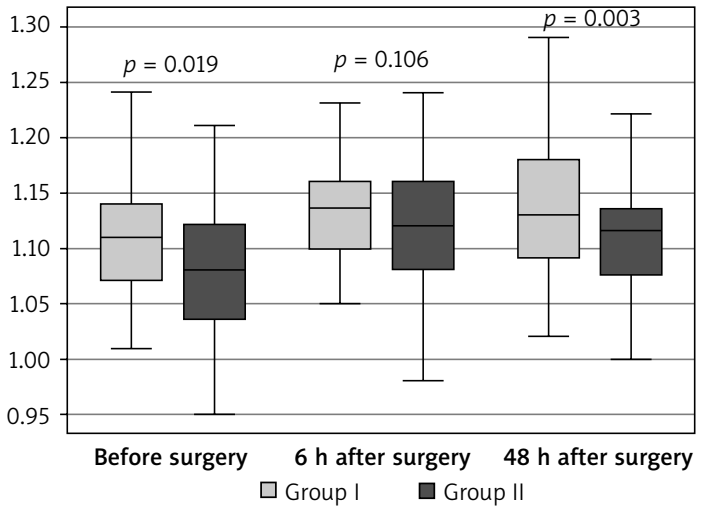

Figure 1. Variable distribution of international normalized ratio in the study patients in group I versus group II, according to the time of measurement

Data presented as median (horizontal line inside box), lower and upper quartile (Q) (lower and upper edge of box, respectively), interquartile range, IQR (distance between quartiles) and values amounting to $Q \pm 1.5^{\star} I Q R$ (whiskers). Number of subjects in group I: $n=40$, in group II: $n=40$. ANOVA without replications was performed for the three time points (relevant p-values shown in plot).

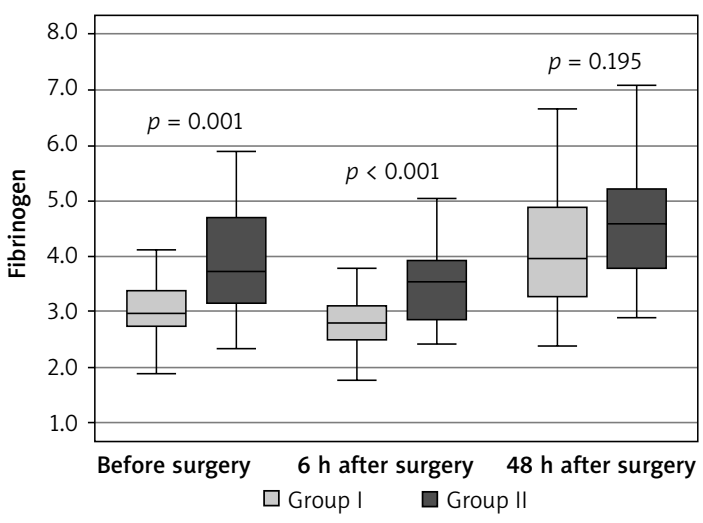

Figure 3. Variable distribution of fibrinogen concentration in study patients in group I versus group II, according to the time of measurement

Results expressed in $\mathrm{g} / \mathrm{l}$. Data presented as median (horizontal line inside box), lower and upper quartile $(Q)$ (lower and upper edge of box, respectively), interquartile range, $I Q R$ (distance between quartiles) and values amounting to $Q \pm 1.5^{*} I Q R$ (whiskers). Number of subjects in group I: $n=40$, in group II: $n=40$. ANOVA without replications was performed for the three time points (relevant $p$-values shown in plot).

the patient's safety. Particular attention should be paid to the group of patients with cardiovascular disease, e.g. permanent atrial fibrillation, who present chronic changes in haemostasis and higher thromboembolic risk [24].

Previous studies on the parameters of the coagulation system in the perioperative period were inconclusive. Correlations between fluctuations in factor VII and the increased perioperative risk of venous thrombosis were postulated, but further studies did not confirm this thesis $[25,26]$.

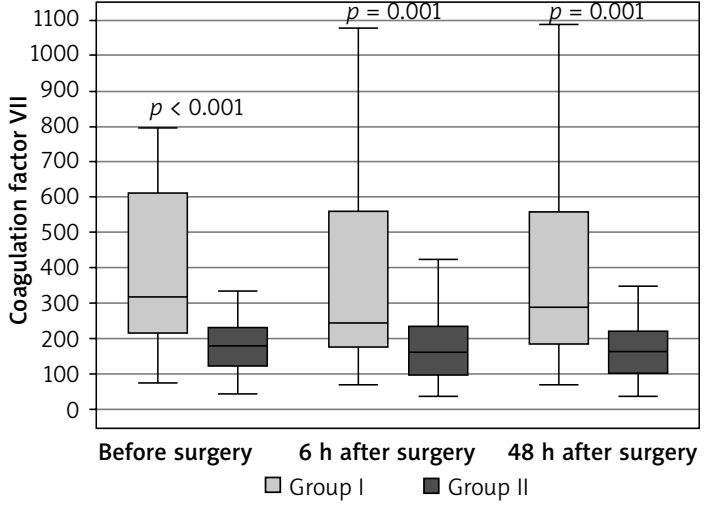

Figure 2. Variable distribution of coagulation factor VII concentration in the study patients in group I versus group II, according to the time of measurement

Results expressed in $\mathrm{ng} / \mathrm{ml}$. Data presented as median (horizontal line inside a box), lower and upper quartile (Q) (lower and upper edge of box, respectively), interquartile range, IQR (distance between quartiles) and values amounting to $Q \pm 1.5^{\star} I Q R$ (whiskers). Number of subjects in group I: $n=40$, in group II: $n=40$. MannWhitney rank-sum test was performed for the three time points (relevant $p$-values shown in plot).

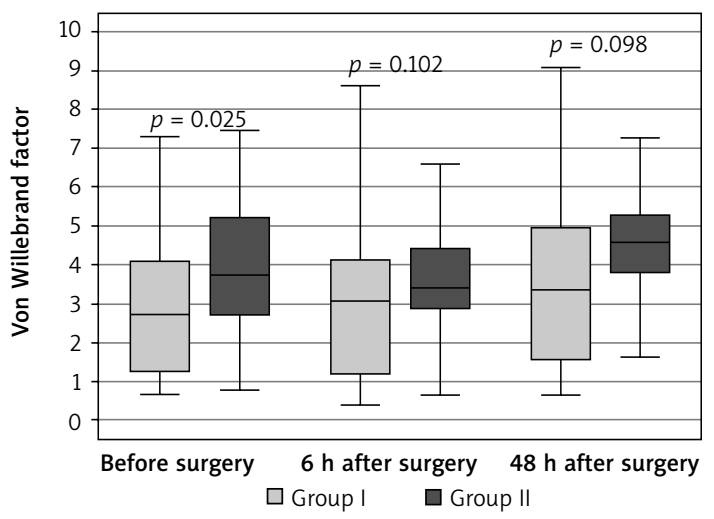

Figure 4. Variable distribution of concentration of von Willebrand factor in study patients in group I versus group II, according to the time of measurement Results expressed in $\mathrm{mU} / \mathrm{ml}$. Data presented as median (horizontal line inside box), lower and upper quartile (Q) (lower and upper edge of box, respectively), interquartile range, IQR (distance between quartiles) and values amounting to $O \pm 1.5^{\star} I Q R$ (whiskers). Number of subjects in group I: $n=40$, in group II: $n=40$. ANOVA without replications was performed for the three time points (relevant $p$-values shown in plot).

The comparison of the length of PT and APTT before and after the surgery did not demonstrate any statistically significant differences [27-29].

Our own study confirmed this tendency, and it also appeared that fibrinogen concentration does not indicate clearly the level of haemostatic system activation. Instead, in our own study it was significantly lower, while Dumitrescu obtained similar results in his study [30].

Ntourakis et al., Schietroma et al. and Vecchio et al. observed increased fibrinogen concentrations following the surgery [28, 29, 31]. 


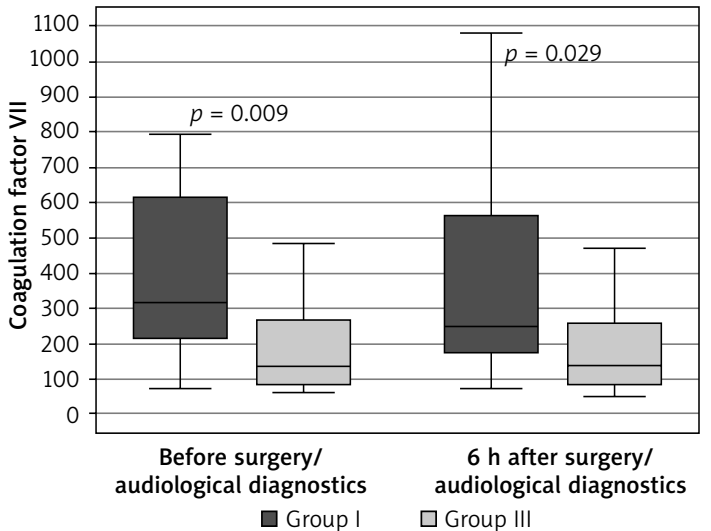

Figure 5. Variable distribution of coagulation factor VII concentration in study patients in group I versus group III not receiving anticoagulants, according to the time of measurement

Results expressed in $\mathrm{ng} / \mathrm{ml}$. Data presented as median (horizontal line inside box), lower and upper quartile $(Q)$ (lower and upper edge of box, respectively), interquartile range, $I Q R$ (distance between quartiles) and values amounting to $Q \pm 1.5^{\star} I Q R$ (whiskers). Number of subjects in group I: $n=40$, in group III: $n=39$. Mann-Whitney rank-sum test was performed for the two time points (relevant $p$-values shown in plot).

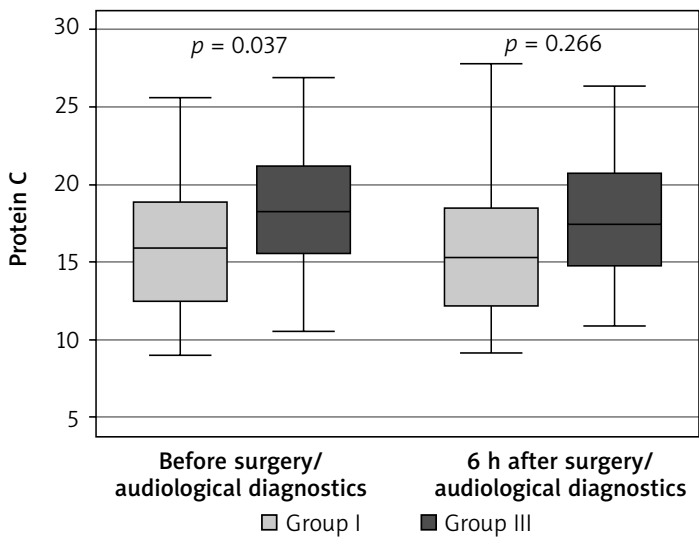

Figure 7. Variable distribution of protein C concentration in study patients in group I versus group III not receiving anticoagulants, according to the time of measurement

Results expressed in $\mathrm{mg} / \mathrm{l}$. Data presented as median (horizontal line inside box), lower and upper quartile (Q) (lower and upper edge of box, respectively), interquartile range, IQR (distance between quartiles) and values amounting to $Q \pm 1.5^{\star} I Q R$ (whiskers). Number of subjects in group I: $n=40$, in group III: $n=39$. ANOVA without replication for the two time points (relevant $p$-values shown in plot).

In our own study we did not find any correlation between the APTT and PT results before and after the surgery, which was confirmed in other studies [27-29].

The analysis of protein C concentrations before and after surgery did not demonstrate any significant differences. Nguyen et al. and Schietroma et al. analysed protein C concentrations before and after surgery, and their studies produced different results [31, 32].

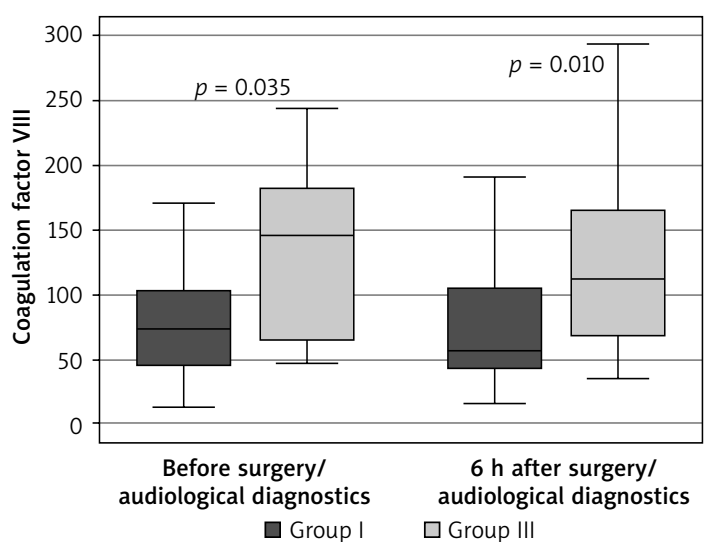

Figure 6. Variable distribution of coagulation factor VIII concentration in study patients in group I versus group III not receiving anticoagulants, according to the time of measurement

Results expressed in $\mathrm{ng} / \mathrm{ml}$. Data presented as median (horizontal line inside box), lower and upper quartile (Q) (lower and upper edge of box, respectively), interquartile range, IQR (distance between quartiles) and values amounting to $Q \pm 1.5^{*} I Q R$ (whiskers). Number of subjects in group I: $n=40$, in group III: $n=39$. Mann-Whitney rank-sum test was performed for the two time points (relevant $p$-values shown in plot).

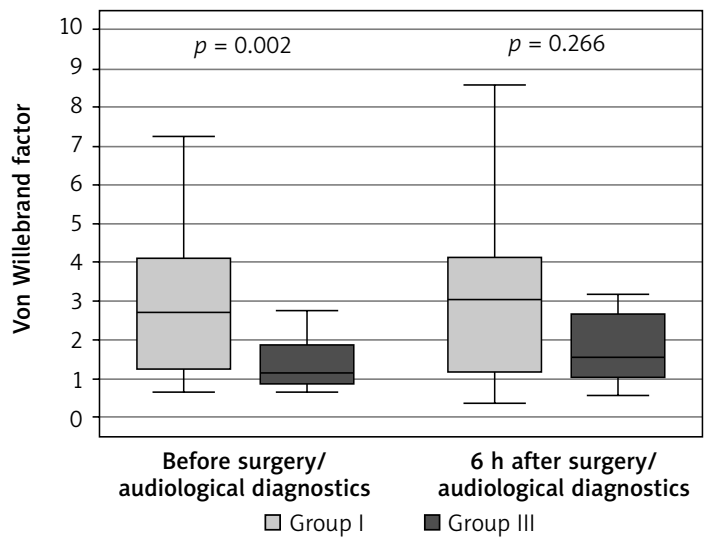

Figure 8. Variable distribution of concentration of von Willebrand factor in study patients in group I versus group III not receiving anticoagulants, according to the time of measurement

Results expressed in $\mathrm{mU} / \mathrm{ml}$. Data presented as median (horizontal line inside box), lower and upper quartile (Q) (lower and upper edge of box, respectively), interquartile range, IQR (distance between quartiles) and values amounting to $Q \pm 1.5^{\star} I Q R$ (whiskers). Number of subjects in group I: $n=40$, in group III: $n=39$. Mann-Whitney rank-sum test was performed for the two time points (relevant $p$-values shown in plot).

The differences in the results of studies monitoring the haemostatic system seem to be significant.

Our study has some limitations. The group of patients is not very large. However, it should be noted that in the available literature there are few studies evaluating so many parameters of the coagulation system during the perioperative period in patients undergoing endoscopic surgery of the paranasal sinuses. It proves that the study pro- 


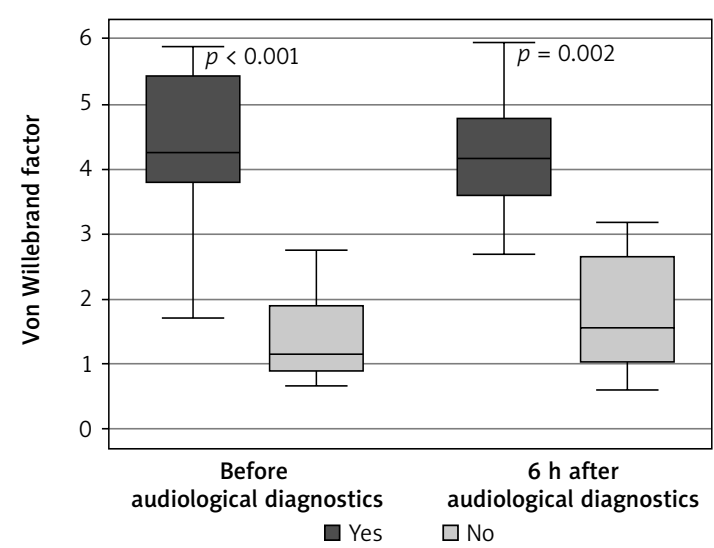

Figure 9. Variable distribution of von Willebrand factor concentration in study patients in group III receiving anticoagulants versus group III not receiving anticoagulants was carried out, according to the time of measurement

Results expressed in $\mathrm{mU} / \mathrm{ml}$. Data presented as median (horizontal line inside box), lower and upper quartile $(Q)$ (lower and upper edge of box, respectively), interquartile range, IQR (distance between quartiles) and values amounting to $Q \pm 1.5^{*} I Q R$ (whiskers). Number of subjects treated with anticoagulants: $n=19$, not taking anticoagulants: $n=20$. ANOVA without replications was performed for the three time points (relevant $p$-values shown in plot).

vides accurate information about haemostasis in such a group of patients.

In conclusion, the correlations between elevated fibrinogen concentration and the administration of low-molecular weight heparin in the perioperative period indicate the increased activity of the haemostatic system in patients receiving antithrombotic treatment. The correlations between the elevated von Willebrand factor levels and use of antiplatelet medications, both before and after audiological diagnostics, without any statistically significant differences between other elements of the coagulation system, may indicate high sensitivity of the von Willebrand factor in deviations regarding the coagulation system parameters. The absence of statistically significant differences in prothrombin concentration and in APTT in the correlations between groups I and II, as well as between groups I and III, indicates that prothrombin and APTT are not useful in presenting the dynamics of the coagulation system in the perioperative period, or in patients using antithrombotic and antiplatelet medications.

\section{Acknowledgments}

Medical University of Lodz Grant number: 50203/7-061-01/502-54-154

\section{Conflict of interest}

The authors declare no conflict of interest.

\section{References}

1. Venkatraman G, Likosky DS, Zhou W, Finlayson SR, Goodman DC. Trends in endoscopic sinus surgery rates in the Medicare population. Arch Otolaryngol Head Neck Surg 2010; 136: 426-30.

2. Khalil HS, Nunez DA. Functional endoscopic sinus surgery for chronic rhinosinusitis. Cochrane Database Syst Rev 2006; 19: CD004458.

3. Amorocho MC, Fat I. Anesthetic techniques in endoscopic sinus and skull base surgery. Otolaryngol Clin North Am 2016; 49: 531-47.

4. Stankiewicz JA, Lal D, Connor M, Welch K. Complications in endoscopic sinus surgery for chronic rhinosinusitis: a 25-year experience. Laryngoscope 2011; 121: 2684-701.

5. Krings JG, Kallogjeri D, Wineland A, Nepple KG, Piccirillo JF, Getz AE. Complications of primary and revision functional endoscopic sinus surgery for chronic rhinosinusitis. Laryngoscope 2014; 124: 838-45.

6. Suzuki S, Yasunaga H, Matsui H, Fushimi K, Kondo K, Yamasoba T. Complication rates after functional endoscopic sinus surgery: analysis of 50,734 Japanese patients. Laryngoscope 2015; 125: 1785-91.

7. Ramakrishnan VR, Kingdom TT, Nayak JV, Hwang PH, Orlandi RR. Nationwide incidence of major complications in endoscopic sinus surgery. Int Forum Allergy Rhinol 2012; 2: 34-9.

8. Thongrong C, Kasemsiri P, Carrau RL, Bergese SD. Control of bleeding in endoscopic skull base surgery: current concepts to improve hemostasis. ISRN Surg 2013; 2013: 191543.

9. Nou M, Laroche JP. Venous thrombo-embolic disease in cancer. Low molecular weight heparin indications. J Mal Vasc 2016; 41: 197-204.

10. Dorobantu M, Bogdan S. Unfractionated heparin or lowmolecular-weight heparin in the elderly. Int J Cardiol 2016; 16: 31602-3.

11. Ortel TL. Perioperative management of patients on chronic antithrombotic therapy. Blood 2012; 120: 4699-705.

12. Al-Eidan FA. Is the incidence trend of heparin-induced thrombocytopenia decreased by the increased use of lowmolecular-weight-heparin? Mediterr J Hematol Infect Dis 2015; 7: e2015029.

13. Douketis JD, Spyropoulos AC, Spencer FA. Perioperative management of anticoagulant therapy: Antithrombotic Therapy and Prevention of Thrombosis, $9^{\text {th }}$ ed: American College of Chest Physicians Evidence-Based Clinical Practice Guidelines. American College of Chest Surgeons Chest 2012; 141 Suppl 2: e326S-50S.

14. Hennekens CH, Sechenova O, Hollar D, Serebruany VL. Dose of aspirin in the treatment and prevention of cardiovascular disease: current and future directions. J Cardiovasc Pharmacol Ther 2006; 11: 170-6.

15. Peters RJ, Mehta SR, Fox KA, et al. Effects of aspirin dose when used alone or in combination with clopidogrel in patients with acute coronary syndromes: observations from the Clopidogrel in Unstable angina to prevent Recurrent Events (CURE) study. Circulation 2003; 108: 1682-7.

16. Kong DF. Aspirin in cardiovascular disorders. What is the optimum dose? Am J Cardiovasc Drugs 2004; 4: 151-8.

17. Nowak-Göttl U, Langer F, Limperger V, Mesters R, Trappe RU. Bridging: perioperative management of chronic anticoagulation or antiplatelet therapy. Dtsch Med Wochenschr 2014; 139: 1301-6.

18. Wigle P, Hein B, Bloomfield HE, Tubb M, Doherty M. Updated guidelines on outpatient anticoagulation. Am Fam Physician 2013; 87: 556-66. 
19. Weitz J. Low-molecular-weight heparins. N Engl J Med 1997; 337: 688-98.

20. Hirsh J, Warkentin TE, Shaughnessy SG, et al. Heparin and low-molecular-weight heparin: mechanisms of action, pharmacokinetics, dosing, monitoring, efficacy, and safety. Chest 2001; 119 (1 Suppl): 64S-94S.

21. Rosenberg RD, Bauer KA. The heparin-antithrombin system: a natural anticoagulant mechanism. Hemostasis and thrombosis: basic principles and clinical practice. $3^{\text {rd }}$ ed. J.B. Lippincott, Philadelphia 1994; 837-60.

22. Kaur S, Kumar A, Lerner RG, Aranow WS. Reversal agents of non-vitamin $\mathrm{K}$ dependent anticoagulants. Arch Med Sci 2016; 12: 1174-8.

23. Kacprzak D, Pawliczak R. Does aspirin-induced oxidative stress cause asthma exacerbation? Arch Med Sci 2015; 11: 494-504.

24. Kusak P, Czarnecka D, Gissel M, Plens K, Butenas S, Undas A. Activated factor IX, factor XI and tissue factor identify patients with permanent atrial fibrillation treated with warfarin who are at risk of ischemic stroke. Arch Med Sci 2016; 12: 1000-7.

25. Milic DJ, Pejcic VD, Zivic SS, et al. Coagulation status and the presence of postoperative deep vein thrombosis in patients undergoing laparoscopic cholecystectomy. Surg Endosc 2007; 21: 1588-92.

26. Milić DJ, Pejić MA, Stojiljković DM, Karanikolić AD, Zivić SS, Visnjić AM. Coagulation status in patients with deep venous thrombosis of the lower extremities. Srp Arh Celok Lek 2003; 131: 201-7.

27. Garg PK, Teckchandani N, Hadke NS, Chander J, Nigam S, Puri SK. Alteration in coagulation profile and incidence of DVT in laparoscopic cholecystectomy. Int J Surg 2009; 7: 130-5.

28. Ntourakis D, Sergentanis TN, Georgiopoulos I, et al. Subclinical activation of coagulation and fibrinolysis in laparoscopic cholecystectomy: do risk factors exist? Int J Surg 2011; 9: 374-7.

29. Vecchio R, Cacciola E, Martino M, Cacciola RR, MacFadyen BV. Modifications of coagulation and fibrinolytic parameters in laparoscopic cholecystectomy. Surg Endosc 2003; 17: 428-33.

30. Dumitrescu G, Januszkiewicz A, Ågren A, Magnusson M Isaksson B, Wernerman J. The temporal pattern of postoperative coagulation status in patients undergoing major liver surgery. Thromb Res 2015; 136: 402-7.

31. Schietroma M, Carlei F, Mownah A, et al. Changes in the blood coagulation, fibrinolysis, and cytokine profile during laparoscopic and open cholecystectomy. Surg Endosc 2004; 18: 1090-6.

32. Nguyen NT, Owings JT, Gosselin R, et al. Systemic coagulation and fibrinolysis after laparoscopic and open gastric bypass. Arch Surg 2001; 136: 909-16. 